Sir,

\section{Reply to P Cackett et al and S Ahmed et al}

Providing patients with tactile feedback from the PAS, to confirm its operation, is perfectly reasonable. Recognition and acknowledgement of the PAS by the operating surgeon or other theatre staff and communicating with the patient, to demonstrate such and ascertain their concerns, is ultimately the desired outcome in this context.

A Mokashi, B Leatherbarrow, J Kincey, R Slater, $\checkmark$ Hillier and S Mayer

Manchester Royal Eye Hospital

Oxford Road

Manchester MI3 9WH

UK

Correspondence: B Leatherbarrow

Tel: + 441612765569

Fax: + 441612726618

E-mail: bollin@mighty-micro.co.uk

Eye (2005) 19, 1025. doi:10.1038/sj.eye.6701725; published online 1 October 2004

Sir,

\section{Bee sting-induced ciliochoroidal detachment}

The sting of members of the order Hymenoptera (eg, bees, wasps, and biting ants) has long been known to cause local and systemic reactions in humans. ${ }^{1}$ Ocular features reported after such stings include conjunctivitis, corneal infiltrates, cataract formation, acute iritis with keratic precipitates, hyphaema, lens subluxation, and rarely optic nerve involvement and retinal damage. ${ }^{2-6}$

We report a case of honeybee sting-induced ciliochoroidal detachment, which to our knowledge, has not been reported earlier.

\section{Case report}

A 25-year-old man presented to the casualty services of our hospital referred by a local ophthalmologist with a history of honeybee sting to the right eye $4 \mathrm{~h}$ back while working in a garden. Patient was complaining of severe pain, photophobia, and diminution of vision in the right eye. The patient was initially found to have a bee sting embedded in the cornea by the local ophthalmologist, which was removed and referred with topical ciprofloxacin $0.3 \%$ drops and prednisolone acetate $1 \%$ drops four hourly. On examination, visual acuity measured finger counting close to face in right eye and 20/20 left eye. The intraocular pressure was $2 \mathrm{mmHg}$ in the right eye and $16 \mathrm{mmHg}$ in the left eye. There was mild diffuse oedema of both upper and lower lids with conjunctival hyperaemia of the right eye. Central corneal oedema with keratic precipitates was present with no obvious embedded sting (Figure 1). The anterior chamber was shallow with +2 cells and +2 flare. Pupil was sluggishly reactive, segmental iris atrophy was present, and the lens showed cataractous changes. Fundus view was precluded by the cataract. B-scan ultrasonography was performed and revealed a smooth, thick dome-shaped membrane with little after movements suggestive of ciliochoroidal detachment (Figure 2a). Ultrasound biomicroscopy confirmed the ciliochoroidal detachment with no evidence of a cyclodialysis cleft (Figure 2b). The left eye showed a normal examination. Pattern visual evoked potential and flash electroretinography was normal in both eyes.

The patient was treated with oral prednisolone, $60 \mathrm{mg}$ daily tapering dose and continued on ciprofloxacin/

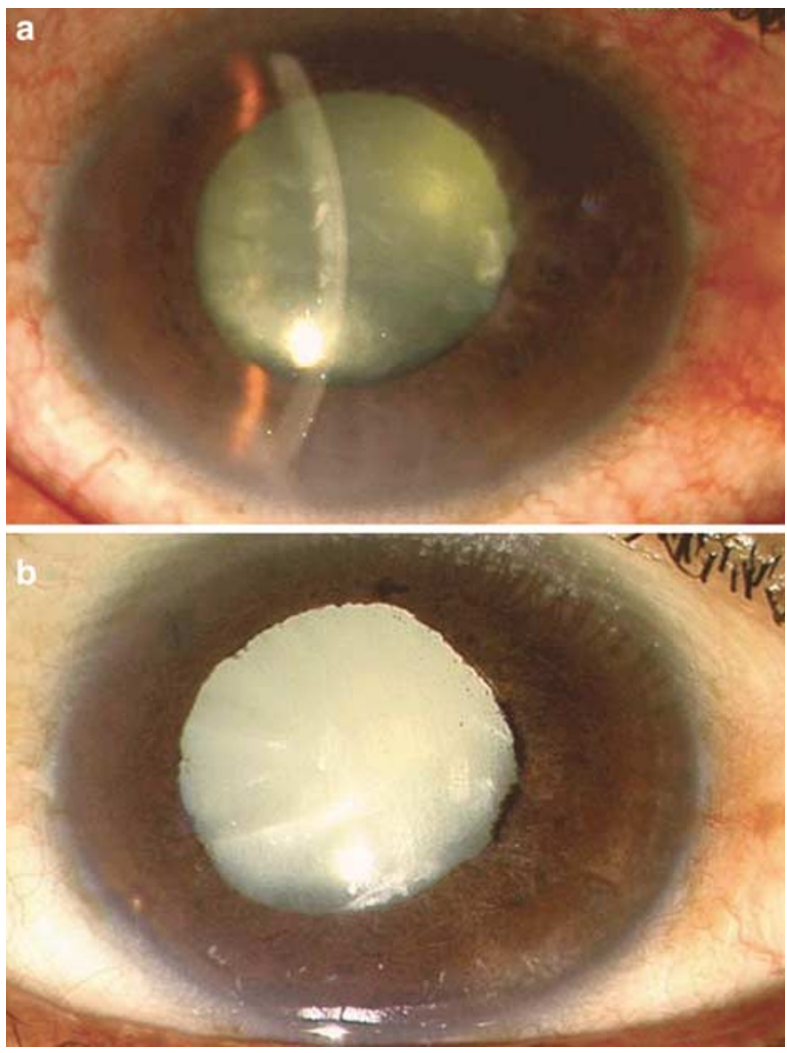

Figure 1 Slit-lamp photograph of right eye: (a) $4 \mathrm{~h}$ after bee sting injury showing corneal oedema, anterior chamber reaction, and cataractous lens and (b) 12 weeks after bee sting injury showing progressed intumscent cataractous lens. 